\title{
Outcomes in patients with inflammatory bowel disease and acute gastrointestinal symptoms who test indeterminate for Clostridioides difficile
}

\author{
Lauren K. Johnson a, Silvia Munoz-Price ${ }^{\mathrm{b}}$, Poonam Beniwal Patel', Amir Patel', Daniel J. Stein', \\ Andres J. Yarurc \\ Medical College of Wisconsin, Milwaukee, WI, USA
}

Abstract

\section{Introduction}

Inflammatory bowel disease (IBD) is a chronic condition of the gastrointestinal tract that can cause patients to suffer from abdominal pain, abdominal cramping, diarrhea, and weight loss.

Department of ${ }^{\mathrm{a}}$ Internal Medicine (Lauren K. Johnson); ${ }^{\mathrm{b}}$ Infectious Diseases (Silvia Munoz-Price); 'Gastroenterology and Hepatology (Poonam Beniwal Patel, Amir Patel, Daniel J. Stein, Andres J. Yarur), Medical College of Wisconsin, USA

Conflict of Interest: None

Correspondence to: Lauren Johnson, Department of Internal Medicine, Medical College of Wisconsin, 3990 John R., Detroit, MI 48201

E-mail: SeidlJohnson@gmail.com

Received 26 September 2021; accepted 13 December 2021; published online 14 February 2022

DOI: https://doi.org/10.20524/aog.2022.0690
Patients with IBD can also present with multiple complications and many require surgery. Clostridioides difficile ( $C$. difficile) infection (CDI) is a disease in which individuals may experience profuse diarrhea with severe cramping and weight loss. Patients with IBD are not only more susceptible to CDI compared to the general population, but may also have worse outcomes, including more severe disease, a higher rate of recurrences, a higher rate of complications, such as need for colectomy, and higher mortality [1-4]. IBD exacerbations and CDI have very similar symptoms and CDI has the potential to precipitate or worsen an underlying IBD exacerbation, causing patients to present with both ailments simultaneously [1,2,4]. Differentiating between IBD exacerbation and CDI can be challenging. Endoscopic examination of the colonic mucosa may not clarify the diagnosis, as the "traditional" colonic pseudomembranes of CDI are not commonly seen in the IBD population [2].

Currently, one of the preferred methods for diagnosing CDI is a nucleic acid amplification test (NAAT) in stool. Because 
NAAT is unable to differentiate between CDI and C. difficile colonization, many hospitals perform a reflex toxin enzyme immunoassay (EIA) to detect circulating toxin following a positive NAAT [5]. However, EIA has a relatively low sensitivity, such that a negative result could indicate either the presence of colonization or a false negative result in the setting of CDI [7].

When $C$. difficile testing yields positive NAAT but negative EIA, practitioners are left with the dilemma of whether to treat these patients for CDI. Polage et al suggest that most EIAnegative patients do not need treatment for CDI, but this refers to the general population [6]. It is well known that IBD patients with a CDI infection are at a higher risk of complications, especially if therapy is delayed [1-4]. Conversely, the unnecessary use of antibiotics could potentially have negative effects on the gut microbiome, associated with greater disease activity in IBD and intestinal infections [8].

To date there have been insufficient data to determine whether the IBD population who test NAAT(+)/EIA(-) should be treated for CDI. Furthermore, the clinical implications of treatment are unknown. The aim of this study was to compare the outcomes of NAAT(+)/EIA(-) IBD patients based on their receipt of CDI treatment.

\section{Patients and methods}

\section{Study design, patients, and setting}

This was a retrospective cohort study that included patients from Froedtert Hospital and the Medical College of Wisconsin, a 735bed tertiary referral center in the Milwaukee area. Ethical approval for the study was obtained from the Institutional Review Board of the Medical College of Wisconsin, Milwaukee, WI. Patients were included if they were 18 years or older, had a confirmed diagnosis of either Crohn's disease (CD) or ulcerative colitis (UC), had an acute exacerbation of gastrointestinal symptoms, were tested for $C$. difficile, and had a NAAT $(+)$ result on the $C$. difficile test.

Patients with a diagnosis of CD or UC who met the inclusion criteria were identified via a local clinical research data warehouse, between the implementation of the reflex test at the institution in January 2017 and the time of data acquisition in July 2019.

\section{Data collected}

Demographic data included age, sex, age at the time of IBD diagnosis, smoking history, body mass index and family history of IBD. Clinical characteristics and IBD phenotype were also abstracted, including clinical disease activity and IBD medications the patient was receiving at the time of testing. Biochemical data included laboratory results at the time of $C$. difficile testing (complete blood count and complete metabolic panel), and C-reactive protein (CRP) levels within 90 days before and 90 days after CDI. Stool cultures, ova and parasite studies, fecal calprotectin, any medical therapies at the time of testing, such as antibiotics, corticosteroids, biologic drugs or immunomodulators, were also collected. Clinical disease activity pre- and post-CDI was recorded using the prospectively collected Harvey Bradshaw index for CD and partial Mayo score for UC, within 6 months of the CDI.

The primary outcome measured was the rate of recurrent CDI, i.e., a test resulting in $\mathrm{NAAT}(+) / \mathrm{EIA}(+)$ for $C$. difficile, within 12 months. Secondary outcomes included changes in IBD therapy within 90 days of the index $C$. difficile testing, complications such as need for bowel resection, and mortality.

\section{Statistical analysis}

Descriptive statistics were used to examine the baseline characteristics of the study population. Continuous variables were compared using Student's $t$-test or the Mann-Whitney $U$ test (for nonparametric variables). Logistic regression was also used. The $\chi^{2}$ test was used to evaluate distributions of categorical variables. A P value of $<0.05$ was considered statistically significant. The analysis was performed using JMP 15 (SAS, North Carolina).

\section{Results}

Among the results of 10,509 C. difficile stool tests, 8703 were NAAT(-) and 1806 were NAAT(+). Among the NAAT(+), 92 tests had been collected from patients who had at some time received ICD diagnosis codes for CD or UC (ICD-10 code K50 or K51). Those 92 NAAT(+) tests had been collected from 71 unique patients. The medical records for the 71 patients were reviewed to confirm the diagnosis of IBD.

The baseline demographic characteristics of the study population are shown in Table 1 . Mean age was 44 years and 39 were female (54\%). Mean age at IBD diagnosis was 31 years, and median time since IBD diagnosis was 9 years. Forty-five patients had CD (63\%) and 26 had UC (37\%). Among the 69 patients for whom smoking status was known, 5 were active smokers (7\%), 22 were former smokers (33\%) and 42 had never smoked (60\%). The 5 active smokers all had CD. Of the total number of tests collected from our cohort, $63 \%$ were obtained from outpatients, $32 \%$ from inpatients, and 5\% from the emergency department. In 33 patients, stool samples were also cultured and examined for ova and parasites, none of which was positive.

\section{Patient characteristics based on EIA results}

Among the 71 patients from our cohort with NAAT(+) testing, 60 tests were EIA negative (84.5\%) and 11 EIA positive (15.5\%). Differences between patients who tested EIA(-) and EIA(+) are shown in Table 1. Patients who tested EIA(+) had significantly higher median levels of pre-CDI CRP compared to those who were $\operatorname{EIA(-)~(1.2~vs.~} 0.5 \mathrm{~g} / \mathrm{dL}, \mathrm{P}=0.02)$, were more likely to be actively smoking (odds ratio [OR] 19, 95\% confidence interval $[\mathrm{CI}] 1.8-198 ; \mathrm{P}=0.0013)$, and had a higher 
Table 1 Baseline variables associated testing NAAT (+) and EIA (-) for C. difficile [Ref: NAAT (+) and EIA (+)]

\begin{tabular}{|c|c|c|c|c|}
\hline Baseline variables & Overall & $\operatorname{EIA}(-)$ & $\operatorname{EIA}(+)$ & P-value \\
\hline Age, mean (SD) & $44(18)$ & $43(18)$ & $49(18)$ & 0.32 \\
\hline Female sex, n (\%) & $39(54)$ & $31(52)$ & $8(67)$ & 0.34 \\
\hline Inpatient testing, $\mathrm{n}(\%)$ & & $22(37)$ & $4(33)$ & 0.83 \\
\hline Active smoker, n (\%) & $5(7.1)$ & $1(2)$ & $3(25)$ & $0.0012^{*}$ \\
\hline Years with IBD, median (IQR) & $9(3-17)$ & $9(4-18)$ & $8(3-16)$ & 0.7 \\
\hline On a biologic drug, $\mathrm{n}(\%)$ & $39(54.2)$ & $33(55)$ & $6(50)$ & 0.75 \\
\hline Diagnosis of CD (vs. UC), n (\%) & $45(62.5)$ & $36(60)$ & $24(40)$ & 0.33 \\
\hline Receiving corticosteroids, $\mathrm{n}(\%)$ & $13(18)$ & $48(80)$ & $11(92)$ & 0.34 \\
\hline Receiving mesalamine, $\mathrm{n}(\%)$ & $12(16.7)$ & $8(13.3)$ & $4(33)$ & 0.09 \\
\hline Receiving immunomodulators, $\mathrm{n}(\%)$ & $17(23.9)$ & $13(22)$ & $4(36.4)$ & 0.3 \\
\hline $\begin{array}{l}\text { Baseline Harvey Bradshaw Index (in CD patients) (pre-CDI), } \\
\text { median (IQR) }\end{array}$ & $2(0-4)$ & $2(0.3-4)$ & $5.5(1-10)$ & 0.17 \\
\hline Partial Mayo score (in UC patients) (pre-CDI), median (IQR) & $1(0-5)$ & $1(0-5)$ & None & N/A \\
\hline Hemoglobin at presentation, mean in $\mathrm{g} / \mathrm{dL}$ (SD) & & $12.5(2)$ & $12(2.7)$ & 0.7 \\
\hline WBC count at presentation, mean in $10^{3} / \mu \mathrm{L}$ (SD) & & $7.8(5.5-11)$ & $12.6(7.8-13.7)$ & $0.024^{*}$ \\
\hline CRP before CDI presentation, median in gr/dL (IQR) & & $0.5(0.13-1.2)$ & $1.2(0.5-11)$ & \\
\hline Platelet count, median in $10^{3} / \mu \mathrm{L}$ (IQR) & & $275(218-366)$ & $264(188-329)$ & 0.79 \\
\hline Albumin, mean in g/dL (SD) & & $4(3.4-4.2)$ & $4.2(3.6-4.3)$ & 0.47 \\
\hline $\mathrm{CRP}$ at baseline, median in $\mathrm{mg} / \mathrm{dL}$ (IQR) & & $0.5(0.1-1.2)$ & $1.1(0.5-4)$ & $0.03^{*}$ \\
\hline $\mathrm{CRP}$ at presentation, median in $\mathrm{mg} / \mathrm{dL}$ (IQR) & & $1.2(0.4-4.8)$ & $4.5(1.4-10.7)$ & 0.19 \\
\hline Fecal calprotectin at presentation, median in $\mu \mathrm{g} / \mathrm{g}$ (IQR) & & $101(23-713)$ & $720(357-1250)$ & 0.09 \\
\hline
\end{tabular}

CD, Crohn's disease; CDI, Clostridioides difficile (C. difficile) infection; EIA, toxin enzyme immunoassay to detect circulating toxin; IBD, inflammatory bowel disease; $I Q R$, interquartile range; NAAT, nucleic acid amplification test for C. difficile in stool; SD, standard deviation; UC, ulcerative colitis; CRP, C-reactive protein

white blood cell count at the time of symptom exacerbation $(\mathrm{P}=0.024)$. Biologic use did not differ between EIA groups. The association of baseline variables with EIA test results in the population are shown in Table 1 . Those variables that were linked to testing NAAT(+)/EIA(-) are shown in Table 2.

\section{Treatments}

\section{Antibiotics}

Of the 60 patients who had an EIA(-) test, 51 were treated with antibiotics (85\%): 44 with vancomycin (86\%), 5 with metronidazole (10\%) and 2 with both vancomycin and metronidazole (4\%). Among the 11 patients whose EIA test was positive, all received antibiotics (100\%): 10 received vancomycin (91\%) and 1 received metronidazole (9\%).

\section{Other medications at time of testing}

Patients with EIA(-) were more likely to be started on a new IBD therapy or corticosteroids when compared to the $\mathrm{EIA}(+)$ group (16 vs. none, $\mathrm{P}=0.012$ ). Fourteen patients received corticosteroids at the time of their acute symptoms and C. difficile testing. Five received budesonide, 7 received prednisone, and 2 received methylprednisolone. At the time of testing 1 patient was started on mercaptopurine, 1 on mesalamine, and 1 on infliximab. Patients started on CDI treatment were less likely to be started on concomitant steroids when compared to those who did not receive CDI treatment (8 [16\%] vs. 4 [44.4\%], $\mathrm{P}=0.047)$.

\section{Outcomes based on EIA results}

\section{Re-testing and recurrences}

Patients who tested EIA(+) were no more likely to require C. difficile testing within 3 months (OR 1.3, 95\%CI 0.13-12.5; $\mathrm{P}=0.84)$. Among $4 \mathrm{EIA}(-)$ patients retested within 3 months, 3 tested NAAT(+)/EIA(-) and 1 tested NAAT $(+) / \mathrm{EIA}(+)$. Of the $11 \mathrm{EIA}(+)$ patients at index testing, 1 retested NAAT $(+) /$ $\operatorname{EIA}(+)$, and none tested NAAT(+)/EIA(-) within 3 months. Patients who tested EIA(+) were as likely to develop a new CDI within 12 months of the index $C$. difficile testing as those that tested EIA(-) initially (OR 0.36, 95\%CI 0.04-3.1; $\mathrm{P}=0.34$ ) (Fig. 1). The association between baseline variables and the development of a CDI within 12 months are shown in Table 3. 


\section{Change in IBD therapy}

Seventeen patients $(23.9 \%)$ underwent a change in their IBD maintenance therapy within 90 days following $C$. difficile testing; this included 14 (23\%) from the EIA(-) group and $3(27 \%)$ from the EIA(+) group. Eight patients were switched to other medications and 7 patients had a medication added to their regimen. EIA(-) patients were no more likely than EIA(+) patients to have a change in IBD therapy (OR 1.18, 95\%CI 0.28-5.0; $\mathrm{P}=0.8$ ).

\section{Complications}

Three patients (4\%) underwent colectomy within 100 days of their acute presentation and $C$. difficile testing; all of them had tested EIA(-). One patient underwent loop ileostomy within 100 days of testing, and that patient had been EIA(+). In addition,

Table 2 Variables associated with testing NAAT(+)/EIA(-)

\begin{tabular}{lccc}
\hline Variables & OR & $95 \%$ CI & P-value \\
\hline Female sex & 0.6 & $0.16-2.3$ & 0.46 \\
\hline Active smoking & 22.1 & $2.05-23.9$ & $<0.001^{*}$ \\
$\begin{array}{l}\text { Diagnosis of Crohn's disease } \\
\text { (vs. ulcerative colitis) }\end{array}$ & 0.56 & $0.14-2.34$ & 0.42 \\
$\begin{array}{l}\text { On biologic therapy } \\
\text { On an anti-tumor necrosis }\end{array}$ & 0.69 & $0.18-2.67$ & 0.59 \\
factor agent & 0.36 & $0.04-3.09$ & 0.34 \\
\hline $\begin{array}{l}\text { On vedolizumab } \\
\text { On corticosteroids }\end{array}$ & 0.4 & $0.05-3.44$ & 0.39 \\
\hline On mesalamine & 0.41 & $0.09-1.88$ & 0.24 \\
\hline On immunomodulator & 0.44 & $0.1-1.72$ & 0.23 \\
\hline
\end{tabular}

$\left.{ }^{*}\right)$ Statistically significant

OR, odds ratio; CI, confidence interval; NAAT, nucleic acid amplification test for Clostridioides difficile in stool; EIA, toxin enzyme immunoassay to detect circulating toxin one patient died within 2 months of the $C$. difficile test, after testing EIA(-). The number of admissions within 30 days from testing was not significantly different between the groups, with 4 EIA(-) patients each being admitted once within 30 days and one $\mathrm{EIA}(+)$ patient being admitted twice within 30 days $(\mathrm{P}=0.78)$.

\section{Outcomes based on CDI treatment}

Among EIA(-) patients, those 51 patients who received CDI therapy were as likely to develop a new CDI within 3 months as were those 9 patients who were not treated. There was one new CDI in the treated group (2\%) and there were no new CDI cases within the untreated group within 3 months of index testing (OR 2.0, 95\%CI 0.36-11.5; $\mathrm{P}=0.096$ ). The patient who had new CDI within 3 months of index EIA(-) test had been treated with vancomycin.

\section{Discussion}

In our retrospective cohort, patients with IBD who presented with acute gastrointestinal symptoms and tested NAAT(+) on C. difficile testing had largely similar outcomes whether toxin EIA was positive or negative. The baseline Harvey Bradshaw Index in CD patients pre-CDI was numerically higher in the EIA(+) group compared to the EIA(-) group; however, the difference was not significant, probably because of the low numbers. We also found that, at our institution, IBD patients who present with gastrointestinal symptoms and test EIA(-) for $C$. difficile are likely to receive antibiotic therapy. However, outcomes were not significantly different between the EIA(-) group based on therapy, although there appeared to be a trend toward higher rates of CDI recurrence in the treated group. This could be explained by the negative effect of antibiotics, or alternatively it could be that patients with more severe baseline IBD are more likely to be treated empirically.

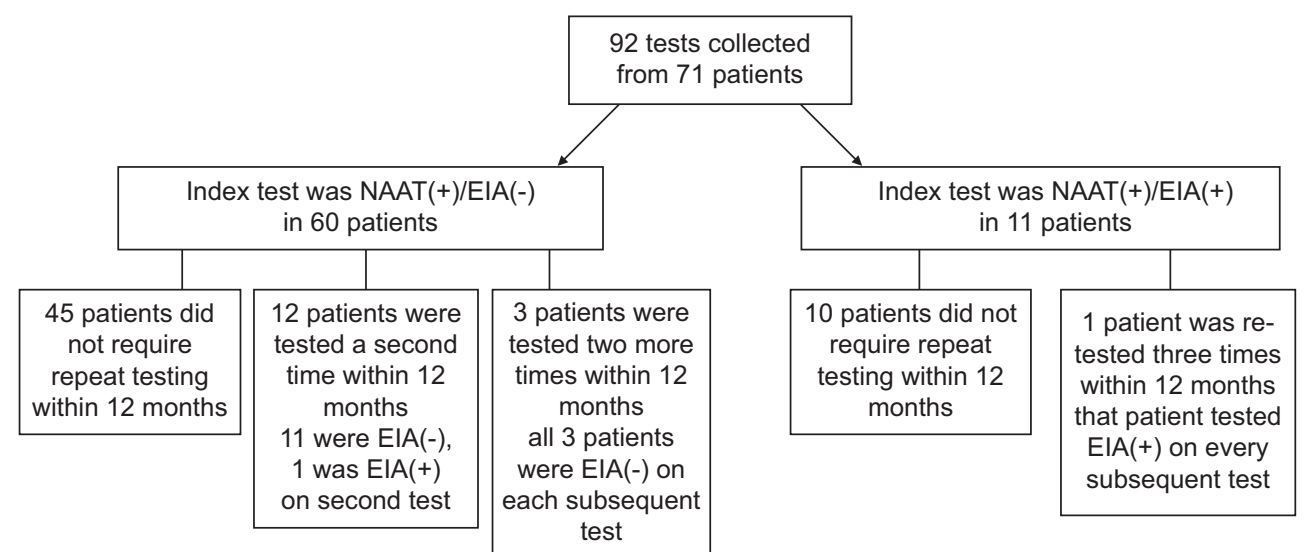

Figure 1 Schematic representation of all $C$. difficile reflex tests performed in our cohort based on whether the initial test was NAAT(+)/EIA(-) or NAAT(+)/EIA(+). Results of any future $C$. difficile testing in either group are also summarized NAAT, nucleic acid amplification test for Clostridioides difficile (C. difficile) in stool; EIA, toxin enzyme immunoassay to detect circulating toxin 
Table 3 Baseline variables associated with $C$. difficile infection within 12 months of testing NAAT (+) for $C$. difficile in patients with inflammatory bowel disease

\begin{tabular}{lccc}
\hline Baseline characteristic & OR & $95 \% \mathrm{CI}$ & $\mathrm{P}$ value \\
\hline $\begin{array}{l}\text { Tested EIA negative for C. } \\
\text { difficile at baseline }\end{array}$ & 0.45 & $0.05-3.9$ & 0.45 \\
$\begin{array}{l}\text { Female sex } \\
\text { Diagnosis of Crohn's disease } \\
\text { (vs. ulcerative colitis) }\end{array}$ & 0.27 & $0.06-1.35$ & 0.1 \\
On biologic therapy & 0.79 & $0.23-2.73$ & 0.71 \\
$\begin{array}{l}\text { On an anti-tumor necrosis } \\
\text { factor agent }\end{array}$ & 1.3 & $0.32-5.4$ & 0.7 \\
$\begin{array}{l}\text { On vedolizumab } \\
\text { On corticosteroids }\end{array}$ & 0.78 & $0.15-4.1$ & 0.77 \\
\hline On mesalamine & 0.36 & $0.04-3.03$ & 0.33 \\
\hline On immunomodulator & 0.51 & $0.17-4.8$ & 0.9 \\
\hline
\end{tabular}

NAAT, nucleic acid amplification test for Clostridioides difficile (C. difficile) in stool; EIA, toxin enzyme immunoassay to detect circulating toxin

Despite the absence of a significant difference in our cohort, these findings are important because this study represents the first investigation into outcomes of IBD patients who have acute gastrointestinal symptoms and indeterminate test results on C. difficile reflex testing in a "real-world" setting. This question is becoming increasingly relevant for clinical practice, as more hospitals adopt the reflex method of testing.

One of the strengths of our study was the fact that it provided a real-world work analysis of how clinicians are approaching the new reflex C. difficile testing in the IBD population. However, the study had several limitations. Bias by indication likely existed, as clinicians were probably more likely to start CDI therapy in those patients with more severe symptoms, fearing the potential development of a CDI complication. Another limitation is the small size of the cohort, leaving the study potentially underpowered to detect significant differences in outcomes upon subgroup analysis.

For IBD patients who test $\mathrm{NAAT}(+) / \mathrm{EIA}(-)$ for C. difficile, clinicians face the issue of whether to prescribe immunosuppressive therapy alone, or start antimicrobial therapy for CDI in conjunction with immunosuppressants. This latter strategy may seem reasonable, especially since delaying therapy of CDI can have catastrophic consequences for patients. However, the unnecessary use of antibiotic treatment can be detrimental, disrupting the structure and function of the gut microbiome and potentially increasing the risk of acquiring other pathogens, creating antibiotic resistance, or even worsening their IBD [8].

Further randomized trials comparing outcomes based on treatment of patients who test NAAT(+)/EIA(-) are warranted. However, until more evidence is available, clinicians need to evaluate patients on a case-by-case basis in order to decide who is more likely to benefit from antibiotic therapy.

In conclusion, in our cohort, treatment for CDI in IBD patients with NAAT(+)/EIA(-) testing did not improve outcomes, or at least was underpowered to detect such a conclusion. There may be a role for administration of antibiotics for IBD patients who test indeterminate on C. difficile testing. However, further study will be needed.

\section{Summary Box}

What is already known:

- Inflammatory bowel disease (IBD) and Clostridioides difficile infection (CDI) have similar presentations

- Patients with IBD are not only more susceptible to CDI compared to the general population, but may also have worse outcomes

- The current preferred laboratory test for CDI is the nucleic acid amplification test (NAAT) for polymerase chain reaction, followed by reflex toxin enzyme immunoassay (EIA) if NAAT is positive

- EIA testing has relatively low sensitivity, making it difficult to decide whether to treat for CDI in special cases such as IBD

\section{What the new findings are:}

- At our institution, patients with IBD were likely to receive antibiotic treatment for CDI if NAAT was positive, even when EIA was negative

- IBD patients with positive NAAT as well as positive EIA were more likely to have elevated white blood cell counts and C-reactive protein levels compared to those for whom the EIA was negative

- Antibiotic treatment for IBD patients who had positive NAAT but negative EIA did not appear to improve outcomes

\section{References}

1. Hashash, JG, Binion, DG. Managing Clostridium difficile in inflammatory bowel disease (IBD). Curr Gastroenterol Rep 2014;16:393.

2. Issa M, Vijayapal A, Graham MB, et al. Impact of Clostridium difficile on inflammatory bowel disease. Clin Gastroenterol Hepatol 2007;5:345-351.

3. Micic D, Yarur A, Gonsalves A, et al. Risk factors for Clostridium difficile isolation in inflammatory bowel disease: a prospective study. Dig Dis Sci 2018;63:1016-1024.

4. Rao K, Higgins PD. Epidemiology, diagnosis, and management of Clostridium difficile infection in patients with inflammatory bowel disease. Inflamm Bowel Dis 2016;22:1744-1754.

5. Swindells J, Brenwald N, Reading N, Oppenheim B. Evaluation of diagnostic tests for Clostridium difficile infection. J Clin Microbiol 2010;48:606-608.

6. Polage CR, Gyorke CE, Kennedy MA, et al. Overdiagnosis of Clostridium difficile infection in the molecular test era. JAMA Intern Med 2015;175:1792-1801.

7. Bartlett JG, Garding DN. Clinical recognition and diagnosis of Clostridium difficile infection. Clin Infect Dis 2008;46(Suppl 1):S12-S18.

8. Isaac S, Scher JU, Djukovic A, et al. Short- and long-term effects of oral vancomycin on the human intestinal microbiota. J Antimicrob Chemother 2017;72:128-136. 